\title{
Potential of a sphingosine 1-phosphate receptor antagonist and sphingosine kinase inhibitors as targets for multiple myeloma treatment
}

\author{
YUKO TANAKA* , SEIICHI OKABE* , KAZUMA OHYASHIKI and AKIHIKO GOTOH \\ Division of Hematology, Tokyo Medical University, Tokyo 160-0023, Japan
}

Received August 2, 2021; Accepted January 18, 2022

DOI: $10.3892 / \mathrm{ol} .2022 .13231$

\begin{abstract}
Sphingosine 1-phosphate (S1P) is a bioactive lipid involved in cancer progression through its binding to S1P receptors (S1PRs). However, the association between multiple myeloma (MM) and S1P is unclear. The current study aimed to investigate the potential anti-cancer effects of fingolimod and sphingosine kinase (SK) inhibitors in myeloma cells and the effects of S1P-induced chemoresistance and neovascularization on MM cell proliferation. MM cell lines were treated with the S1PR1 antagonist fingolimod and the SK inhibitors ABC294640 and SK1-I, after which cell proliferation was measured. Protein expression was also assessed under each condition using immunoblotting. Serum S1P levels in patients with MM, monoclonal gammopathy of undetermined significance and healthy volunteers were assessed. Human umbilical vessel cells (HUVECs) were co-cultured with anti-S1P agents to assess the effect on cell migration. All treatments suppressed myeloma cell proliferation and caspase-3-mediated apoptosis by suppressing S1P activity. These findings suggest that S1P activation is associated with proliferation and survival for MM cells. S1P attenuated the proteosome inhibitor (PI) effect, while the anti-S1P agents recovered the effect. In addition, S1P promoted the migration and proliferation of HUVECs, whereas the S1P inhibitors reduced the influence of S1P. This study highlights the therapeutic potential of anti-S1P agents for MM treatment. Inhibition of S1P function may overcome resistance to PI developed by myeloma cells and inhibit the changes to the bone marrow microenvironment via neovascularization.
\end{abstract}

Correspondence to: Dr Yuko Tanaka, Division of Hematology, Tokyo Medical University, 6-7-1 Nishi-shinjuku, Shinjuku-ku, Tokyo 160-0023, Japan

E-mail: yukois9@tokyo-med.ac.jp

*Contributed equally

Key words: sphingosine 1-phosphate, sphingosine 1-phosphate receptor, fingolimod, sphingosine kinase, fingolimod, multiple myeloma, proteasome inhibitor, bone marrow microenvironment

\section{Introduction}

The survival of patients with multiple myeloma (MM) has been remarkably extended because of the development of new proteasome inhibitors (PIs) and immunomodulatory drugs $(1,2)$. However, most patients treated with these drugs ultimately relapse owing to the development of chemotherapeutic resistance in MM cells (3).

Sphingosine-1 phosphate (S1P) was originally identified as a bioactive lipid and has been reported to be involved in the regulation of various physiological cell functions, such as cell proliferation, apoptosis, and angiogenesis (4). A molecule of S1P contains a ceramide backbone; sphingosine (SP) is catalyzed by two enzymes, namely, sphingosine kinase 1 (SK1) and sphingosine kinase 2 (SK2). S1P exerts its activity, both inside and outside the cell membrane, by interacting with a G protein-coupled S1P receptor (S1PR) on the cell membrane. Five isotypes of S1PR have been identified (S1PR1-S1PR5), and their respective functions have been reported $(5,6)$. Several studies have reported that S1P influences cancer progression (7-10). Indeed, high activity of S1P and its synthetases, SKs, combined with high expression of specific S1PR isotypes has been reported in numerous cancer types $(8,10)$. Fingolimod is an S1PR1 receptor antagonist that was recently adopted as a therapeutic drug for multiple sclerosis, and its efficacy on various tumors in inducing apoptosis and reducing angiogenesis has been previously reported (11). Moreover, small-molecule SK inhibitors with anti-cancer potential against cancer cell survival and proliferation have been identified (12-17). SK1-I is synthesized as a sphingosine analog and specifically inhibits SK1. It reportedly inhibits growth and survival by inducing apoptosis in leukemia cells (12). ABC294640 is an SK2-specific inhibitor. This compound reportedly inhibits tumor proliferation and migration by promoting autophagic cell death $(13,14)$.

However, the role of S1P in regulating myeloma cell proliferation is unclear. We hypothesized that the bioactivity of S1P affects myeloma cell proliferation or the acquisition of chemotherapeutic resistance. Thus, targeting S1PR or the enzymes involved in S1P biosynthesis may serve as a novel therapeutic strategy for MM. To test this hypothesis, we evaluated the potential anti-cancer effects of fingolimod and SK inhibitors in myeloma cells and investigated the effects of S1P-induced chemoresistance and neovascularization on MM cell 
proliferation. Moreover, we evaluated circulating S1P levels in the serum of patients with MM and monoclonal gammopathy of undetermined significance (MGUS) to identify candidate biomarkers capable of detecting disease progression of MM or its advancement to a later disease stage.

\section{Materials and methods}

Cell lines and primary myeloma cell culture. The human myeloma cell lines RPMI8226, MM1S, MM1R, and human umbilical vein endothelial cells (HUVECs) were purchased from ATCC. Primary myeloma cells were derived from the peripheral blood of two patients diagnosed with plasma cell leukemia (PCL). Mononuclear cells were separated using Lymphosepar (Immuno-biological Laboratories Co.). All cells were cultured in RPMI-1640 medium supplemented with $10 \%$ heat-inactivated fetal bovine serum and $1 \%$ penicillin/streptomycin in a humidified incubator containing $5 \% \mathrm{CO}_{2}$ at $37^{\circ} \mathrm{C}$. The study protocol was approved by the Institutional Review Board of Tokyo Medical University (no. SH2408). Written informed consent was obtained from all patients in accordance with the tenets of the Declaration of Helsinki.

Cell proliferation assay. Cell proliferation was assessed using 3-[4,5-dimethylthiazol-2-yl]-2,5 diphenyl tetrazolium bromide assay with the Cell Euros Kit-8 (Dojindo Molecular Technologies) in accordance with the manufacturer's protocol.

Immunoblotting. Immunoblot analysis was performed as previously described (18). After appropriate treatment, the cells were washed with ice-cold PBS twice and lysed with a radioimmunoprecipitation assay lysis buffer. Forty micrograms of total protein extract was separated on 4-20\% polyacrylamide gels and electro-transferred to a polyvinylidene difluoride membrane. Thereafter, the membrane was probed using the primary antibodies of interest at 1:1,000 dilutions for $1 \mathrm{~h}$ at $25 \pm 1^{\circ} \mathrm{C}$. We then used the Amersham ECL chemiluminescence kit (GE Healthcare) in accordance with the manufacturer's instructions. We used the following primary antibodies: anti-phospho-S6 ribosomal protein (Ser235/236), anti-cleaved caspase-3, and anti-poly-ADP-ribose polymerase (PARP), purchased from Cell Signaling. Antibodies against MAPK ERK1 and $\beta$-actin were purchased from Santa Cruz Biotechnology. The experiments were carried out in three independent replicates. Protein band intensity was evaluated using ImageJ software (National Institutes of Health).

Reverse-transcription PCR. Reverse-transcription PCR (RT-PCR) was performed as previously described (1). Total RNA was extracted form MM cells, HUVECs, and primary patient samples using the RNA queous ${ }^{\circledR}-4 \mathrm{PCR}$ kit (Life Technologies Japan, Ltd.). The RNA concentration was determined spectrophotometrically. Next, 82 ng RNA was used to synthesize cDNA using a first-strand cDNA synthesis kit (OriGene Technologies) under the following reaction conditions: 1 cycle at $22^{\circ} \mathrm{C}$ for $5 \mathrm{~min}, 1$ cycle at $42^{\circ} \mathrm{C}$ for $30 \mathrm{~min}$, and then 1 cycle at $85^{\circ} \mathrm{C}$ for $5 \mathrm{~min}$, followed by a hold at $4^{\circ} \mathrm{C}$. RT-PCR was performed using a PCR Master Mix (Promega Corporation) and the Roche Light Cyber 2.0 detection system
(Roche Diagnosis Gmbh). Thermocycling conditions were as follows: $95^{\circ} \mathrm{C}$ for $5 \mathrm{~min}$, then 40 cycles at $95^{\circ} \mathrm{C}$ for $30 \mathrm{sec}$, $55.5^{\circ} \mathrm{C}$ for $30 \mathrm{sec}$, and $72^{\circ} \mathrm{C}$ for $1 \mathrm{~min}$. The primer sequences were as follows: GAPDH forward, 5'-ACCACAGTCCATGCC ATCAC-3' and GAPDH reverse, 5'-TCCACCACCCTGTTG CTGTA-3'. The GAPDH primer was purchased from Life Technology Japan, Ltd. The specific PCR primers of S1PR1, S1PR2, S1PR3, S1PR4, S1PR5, SK1, and SK2 were purchased from Santa Cruz Biotechnology. The information of the sequences of these primers could not be provided from the company then the sequences are not publicly available.

Lentiviral SK1 and SIPRI ShRNA. We purchased lentiviral vector shRNAs of shS1PR1, SK1, and scramble shRNA from Vector Builder Japan. The method of lentiviral vector transduction into RPMI8226 cells followed the Addgene protocol (http://www.addgene.org/tools/protocols/plko\#E). We used Polybrene for enhancing lentiviral transduction with cells, the concentration of Polybrene $5 \mu \mathrm{g} / \mathrm{ml}$ and $2 \mu \mathrm{l}$ shRNA was mixed and cultured overnight. The effect of shRNA knockdown for SK1 and S1PR1 expression was estimated by real time PCR and immunoblotting. Total RNA extraction and cDNA synthesis did the same method as previously. Real time PCR was performed using a Fast Strand Essential DNA Green Master and Roche Light Cyber 2.0 detection system (Roche Diagnosis Gmbh). Thermocycling conditions were as follows: $95^{\circ} \mathrm{C}$ for $10 \mathrm{~min}$, then 45 cycles at $95^{\circ} \mathrm{C}$ for $10 \mathrm{sec}$, $60^{\circ} \mathrm{C}$ for $30 \mathrm{sec}$. The primer sequences were as follows: S1PR1 forward, 5'-GGCTATGTTGAGTACGTAGGCTGTG-3' and S1PR1 reverse, 5'-TCCCGCTTACATGGAAACTTTG-3', SphK1 forward, 5'-CTGGCAGCTTCCTTGAACCAT-3' and SphK1 reverse, 5'-TGTGCAGAGACAGCAGGTTCA-3' (Takara Bio). The immunoblotting technique was the same method as previous mentioned. Anti-S1PR1 and SphK1 rabbit polyclonal antibodies were purchased from Proteintech ${ }^{\circledR}$. The membrane was probed using the primary antibodies of interest at 1:300 dilutions. The shRNA knockdown cells were cultured with carfilzomib and the suppressive ability for cell proliferation was assessed by caspase-3/7 activity. We used the kit, Caspase-Glo ${ }^{\circledast}$ 3/7 Assay (Promega Corporation).

Chemotaxis assay. The chemotaxis assay for HUVECs was performed using a Boyden chamber with an $8-\mu \mathrm{m}$ pore size (Corning, Inc.) (19). In the upper chamber, we seeded HUVECs. In the lower chamber, indicated materials (supernatant of cell line, S1P and anti-S1P agents) were added with medium (0.2\% FBS DMEM). The cells were incubated at $37^{\circ} \mathrm{C}$ for $4 \mathrm{~h}$ in humidified air with $5 \% \mathrm{CO}_{2}$. Non-migrated cells were removed by a cotton swab, and migrated cells were stained by May-Giemsa method. The stained migrated cells were counted by microscopic $x 100$ field of vision in three random fields. The cell number was average of three random fields.

Assessment of serum SIP concentration among MM patients, MGUS patients, and healthy adults via ELISA. We determined serum S1P levels in 13 patients who were newly diagnosed with MM, in five patients with MGUS, and age-matched 16 healthy volunteers. The specimens were harvested in 2013, and patients and healthy volunteers provided informed consent to participate in the study (approved no. 'SH2408'). However, 
sample size determination, randomization, and blinding were not performed as some people could not consent when we planned to design another clinical study for S1P measurement. Furthermore, the serum S1P levels were assessed using an ELISA Sphingosine 1-phosphate Assay Kit (Echlon, Inc.).

Statistical analysis. The Mann-Whitney U-test was used to estimate serum S1P levels among MM patients, MGUS patients, and healthy volunteers, and between MM patients with or without symptoms. The Student's t-test was used to assess the effects of drug treatment in comparison with the control group. $\mathrm{P}<0.05$ was considered significant. All data were analyzed using IBM SPSS statistic ver.28 and Prism8.

\section{Results}

mRNA expression levels of SK1, SK2 and SIPR1-S1PR5 in HUVECs and MM cells. We performed RT-PCR to confirm the mRNA expression of both SK1 and SK2 in the following samples: HUVECs, primary myeloma cells from two patients with PCL, and three MM-derived cell lines, RPMI8226, MM1.S and MM1.R (Fig. 1A). As S1P exerts its effects by binding to S1PRs, we examined the mRNA expression of the five S1PR isotypes (S1PR1-S1PR5) to determine their expression patterns in our cell lines. All tested cell lines expressed S1PR1 mRNA, the target of fingolimod, but not S1PR4 mRNA. S1PR2 mRNA was expressed in all cells, except for one of the PCL samples (sample A). Finally, we observed S1PR3 mRNA expression in MM1.S, MM1.R, PCL sample A, and HUVECs (Fig. 1B). It has been reported that S1PR1, S1PR2 and S1PR3 are expressed in almost all tissues and organs; conversely, S1PR4 and S1PR5 are expressed mainly in lymphoid tissue (20). Our results are consistent with these previous observations.

Serum SIP levels increased in patients with MM. We next determined the serum levels of S1P in MM patients using ELISA. The results showed that the serum concentration of S1P was higher in MM patients and MGUS patients than in healthy age-matched controls. The difference between MM and healthy age-matched controls was significant $(\mathrm{P}<0.05$; Fig. 2A). Moreover, among MM patients with anemia as a complication, the median serum S1P level was significantly lower than that in the group without anemia $(\mathrm{P}<0.05$; Fig. $2 \mathrm{~B})$. Regarding other complications, including the co-occurrence of bone disease, hypercalcemia, and renal dysfunction, we did not observe a significant change in serum $\mathrm{S} 1 \mathrm{P}$ levels $(\mathrm{P}<0.05$; Fig. 2C-F). The group profile (MM, MGUS, and healthy control) and the number of patients with MM symptoms are shown in Fig. 2F.

Fingolimod, SKI-I, and ABC294640 inhibit the proliferation of $M M$ cell lines. We investigated the effect of fingolimod, SKI-I, and ABC294640 on the proliferation of MM cells. All agents inhibited the growth of RPMI8226, MM1.S, and MM1.R cell lines at concentrations of 5 and $10 \mu \mathrm{M}(\mathrm{P}<0.05$; Figs. 3A and S1A and B). Thereafter, we investigated the effects of the three anti-S1P agents on intracellular signaling in RPMI8226 cells. RPMI8226 cells were treated with the indicated anti-S1P agents for $24 \mathrm{~h}$. At high concentrations, the three molecules inhibited the phosphorylation of the S6 ribosomal
A
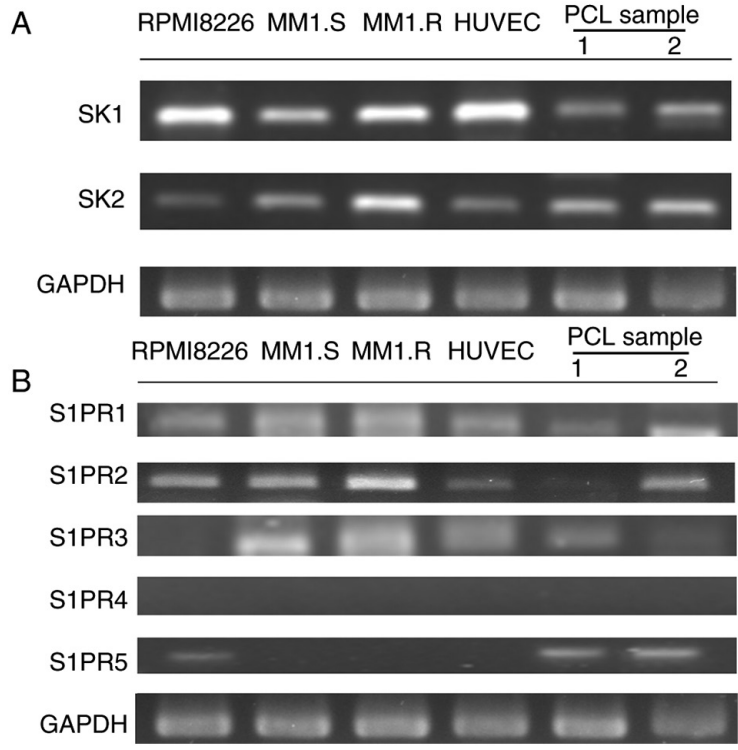

Figure 1. Expression of S1PR and SPK mRNA in multiple myeloma cell lines, HUVECs and primary samples. (A) SPK1 and SPK2 mRNA expression, and (B) the expression of five isotype S1P receptors (S1PR1-S1PR5) were assessed. HUVECs, human umbilical vessel cells; PCL, plasma cell leukemia; S1PR, sphingosine 1-phosphate receptor; SK, sphingosine kinase.

protein. At the same concentration, we observed induction of PARP and cleaved caspase-3 (Fig. 3B). To confirm the relation between inhibition of SK or S1PR function and myeloma cell proliferation, we assessed the effect of SK1 and S1PR1 mRNA knockdown. Western blotting revealed that caspases were related to cell apoptosis induced by SKs and S1PRs, and therefore, we estimated and confirmed caspase activity. We concluded that suppression of the S1P pathway induced caspase-mediated myeloma cell death. We next evaluated whether caspase-3/7 activity was affected by shRNA and culturing with carfilzomib. shRNA knockdown was performed by lentiviral infection to RPMI8226, and we confirmed the transfection efficiency by RT-qPCR and immunoblotting. The result of immunoblotting and RT-qPCR showed the knock down efficiency for both protein and DNA of S1PR1 and SK1 by shRNA (Fig. S1C and D). RPMI8226 that was performed bySK1 or S1PR1 knockdown using shRNA showed a significantly higher caspase activity than did non-knockdown cells. When carfilzomib was added to the culture, the caspase-3/7 activity was higher than that in knockdown cells without carfilzomib. These results demonstrated that S1PR1 and SK1 were concerned with myeloma cell survival and S1P interfered the cytotoxic effect of proteasome inhibitor for myeloma cell. (Fig. S1E and F).

Use of fingolimod, SKI-I, and ABC294640 in combination with carfilzomib protease inhibitor enhanced anti-tumor activity in MM cell lines and primary cells. Three agents were further assessed in combination with the PI carfilzomib in RPMI8226 MM cells. Because we observed more effective for cell reduction at $5 \mathrm{nM}$ carfilzomib than at $2 \mathrm{nM}$ carfilzomib previously (Fig. S1F), we evaluated the impact of the combination of $5 \mathrm{nM}$ carfilzomib with fingolimod, SKI-I, or ABC294640 on cell growth at the concentration that suppressed MM cell growth (Figs. 3A and S1A and B). The results showed that fingolimod $(5 \mu \mathrm{M})$, SKI-I $(2.5 \mu \mathrm{M})$, 
A

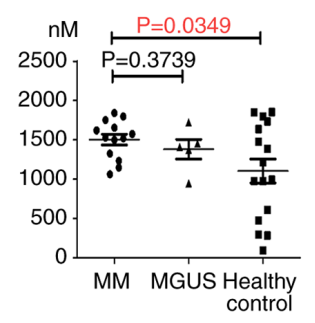

B

\begin{tabular}{|c|c|c|c|c|}
\hline & & $\underset{(n=13)}{M M}$ & $\begin{array}{c}\text { MGUS } \\
(n=5)\end{array}$ & $\begin{array}{l}\text { Normal } \\
(n=16)\end{array}$ \\
\hline Male// & emale & $3: 10$ & $2: 3$ & $12: 4$ \\
\hline Age $(r$ & edian) & 63 & 67 & 62 \\
\hline S1P ( & nedian; $\mathrm{nM}$ & 1503.4 & 1380.5 & 1103.0 \\
\hline $\mathrm{N}$ & Anemia B & one diseas & $\begin{array}{l}\text { Hyper- } \\
\text { calcemia }\end{array}$ & $\begin{array}{c}\text { Renal } \\
\text { dysfuction }\end{array}$ \\
\hline Yes & 9 & 4 & 2 & 2 \\
\hline No & 4 & 9 & 11 & 11 \\
\hline
\end{tabular}

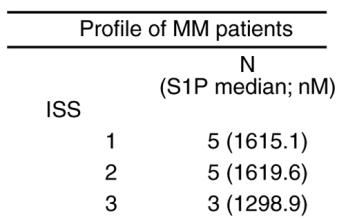

\begin{tabular}{rr} 
M protein & \\
$\operatorname{IgG}$ & 7 \\
IgA & 2 \\
Bence-Jones & 4 \\
Kappa & 8 \\
Lambda & 5 \\
\hline
\end{tabular}
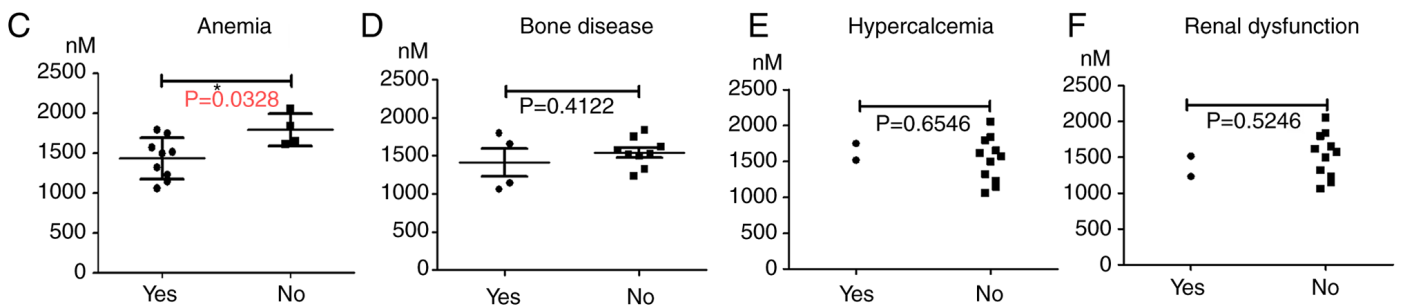

Figure 2. Serum S1P levels as measured by ELISA. (A) Serum S1P levels were determined in patients with MM and compared with patients with MGUS $(\mathrm{P}=0.0379)$ and healthy controls $(\mathrm{P}=0.0349)$. (B) The clinical feature and median serum S1P level of each group, the number of MM patients with or without complications and profile of MM patients including ISS (median serum S1P level) and M protein types. Serum S1P levels in patients with MM and various complications, including (C) anemia, (D) bone disease, (E) hypercalcemia and (F) renal dysfunction, were compared with those individuals that did not demonstrate these complications. Red indicates significant P-values. ISS, international scoring system; MGUS, monoclonal gammopathy of undetermined significance; MM, multiple myeloma; S1P, sphingosine 1-phosphate.

A

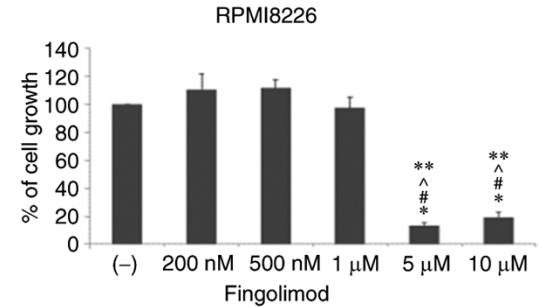

B

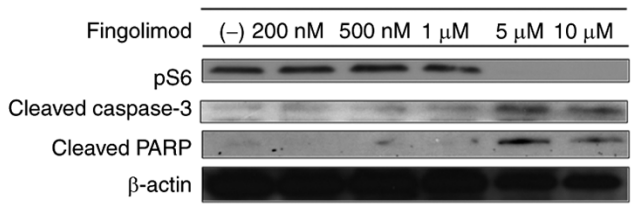

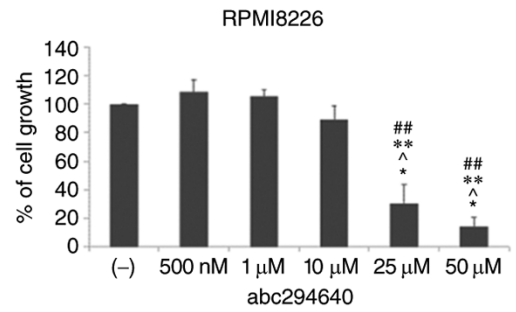

RPMI8226

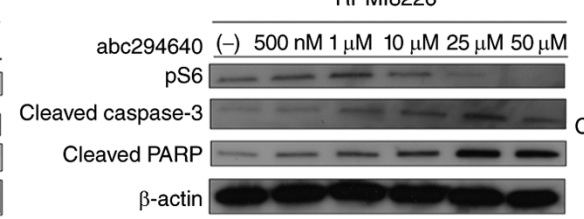

RPMI8226

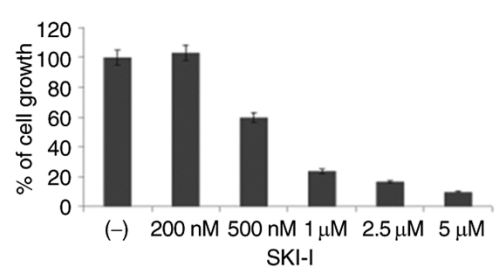

RPMI8226

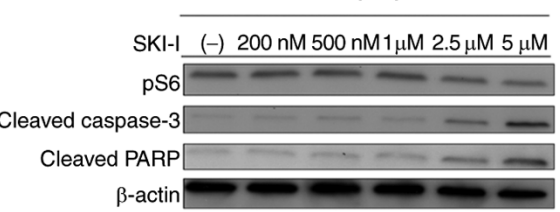

Figure 3. Effects of anti-sphingosine 1-phosphate agents on the growth of MM cell lines. (A) Fingolimod, SKI-I and ABC294640 suppressed the growth of the MM cell line (RPMI8226). (B) immunoblotting analysis was performed to measure the total protein extract after $24 \mathrm{~h}$. ${ }^{*} \mathrm{P}<0.05 \mathrm{vs}$. control; ${ }^{\# P} \mathrm{P}<0.05 \mathrm{vs} .200 \mathrm{nM}$; ${ }^{\wedge} \mathrm{P}<0.05$ vs. $500 \mathrm{nM} ;{ }^{* *} \mathrm{P}<0.05$ vs. $1 \mu \mathrm{M}$ and ${ }^{\# \#} \mathrm{P}<0.05$ vs. $10 \mu \mathrm{M}$. MM, multiple myeloma; PARP, poly (ADP-ribose) polymerase; SK, sphingosine kinase.

and $\mathrm{ABC} 294640(25 \mu \mathrm{M})$ in combination with carfilzomib significantly increased cell growth compared to $5 \mathrm{nM}$ carfilzomib treatment alone (Fig. 4A). We then assessed the effect of these combinations on the proliferation of PCL sample A. Consequently, the combination of fingolimod $(3 \mu \mathrm{M})$, SKI-I $(2.5 \mu \mathrm{M})$, or ABC294640 $(25 \mu \mathrm{M})$ with $5 \mathrm{nM}$ carfilzomib synergistically inhibited the growth of primary MM cells (Fig. 4B). In each bar plot of Fig. 4A and 4B, the mean and SD of three independent replicates are shown.

We next investigated the effects of the three anti-S1P agents in combination with carfilzomib on intracellular signaling in RPMI8226 cells. RPMI8226 cells were treated with fingolimod, SKI-I, or ABC294640, with or without carfilzomib, for $24 \mathrm{~h}$. We observed that combinatorial treatment exerted the same effect as that observed with the three agents alone, resulting in similar protein expression patterns (Figs. 3B and 4D). Notably, the combinatorial treatment inhibited the S6 ribosomal protein more strongly than carfilzomib monotherapy, which was consistent with the observed synergistic cell growth inhibition. Moreover, we observed increased cleaved PARP activation upon treating cells with the same amount of inhibitor used for cells harvested from patients with PCL (Fig. 4C).

SIP attenuates the PI-mediated anti-tumor effect in MM cells, which is recovered by co-treatment with anti-SIP agents. After confirming that the serum S1P level was higher in patients with MM than in healthy controls, we examined the effect of the addition of exogenous S1P to RPMI8226 cells co-cultured 
A

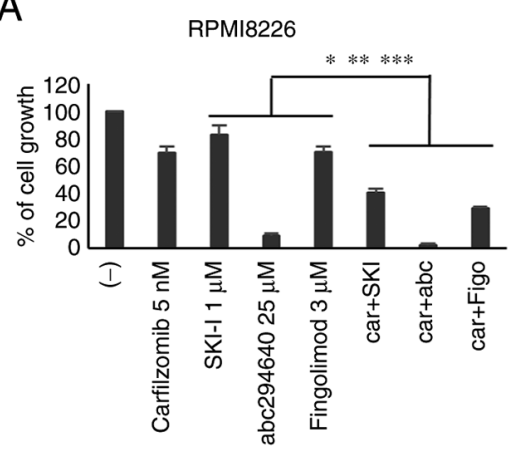

B

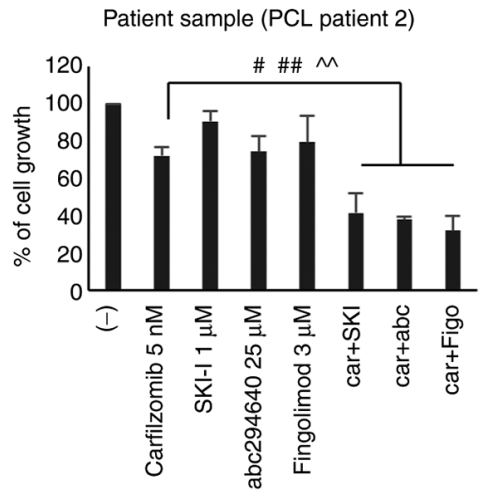

C

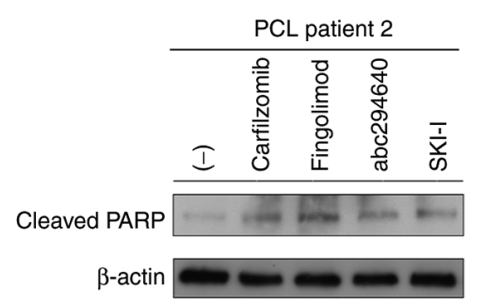

D

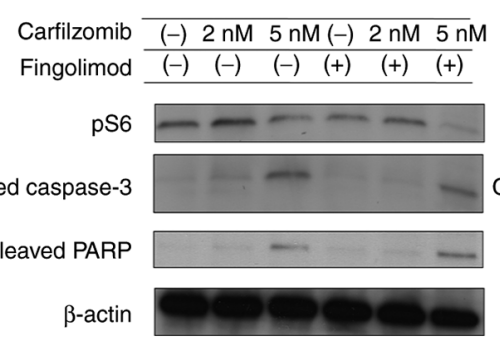

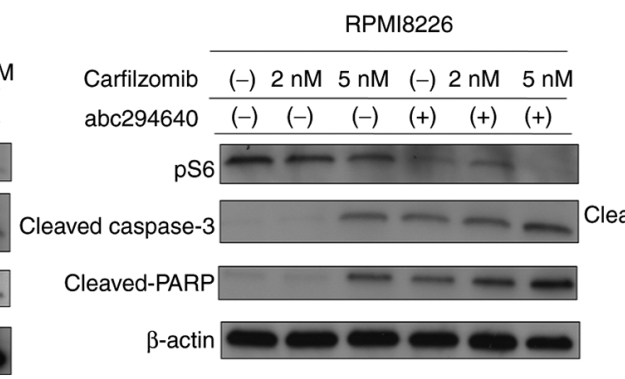

RPMI8226

Carfilzomib (-) $2 \mathrm{nM} 5 \mathrm{nM}(-) 2 \mathrm{nM} 5 \mathrm{nM}$ $\begin{array}{lllllll}\operatorname{abc} 294640 & (-) & (-) & (-) & (+) & (+) & (+)\end{array}$

pS6 - -
Carfilzomib \begin{tabular}{c} 
RPMI8226 \\
\cline { 2 - 4 } \\
\cline { 3 - 4 }
\end{tabular}

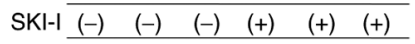

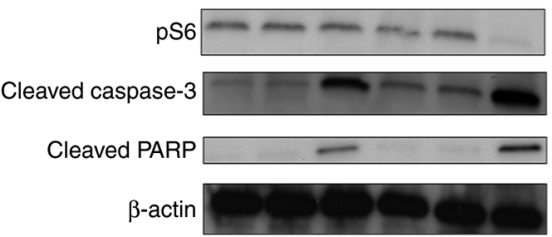

Figure 4. Effect of fingolimod, SK-1I and ABC294640 in combination with carfilzomib. (A) RPMI8226 or (B) PCL patient cells were used for assessment. Images represent the indicated total protein extract examined by immunoblot analysis for (D) fingolimod, ABC294640 and SKI-I, and (C) cleaved PARP. ${ }^{*} \mathrm{P}<0.05$ vs. car+SKI; ${ }^{* *} \mathrm{P}<0.05$ vs. car+abc; ${ }^{* * * *} \mathrm{P}<0.05$ fingolimod $3 \mu \mathrm{M}$ vs. car+Figo; ${ }^{\#} \mathrm{P}<0.05$ vs. car+SKI; ${ }^{\# \#} \mathrm{P}<0.05$ vs. car+abc and ${ }^{\wedge} \mathrm{P}<0.05$ vs. car+Figo. abc, ABC294640; car, carfilzomib; Figo, fingolimod; PCL, plasma cell leukemia; PARP, poly(ADP-ribose) polymerase; SKI, SKI-I.

with carfilzomib alone or through sequential addition of fingolimod, SKI-I, or ABC294640. As shown in Fig. 5A, the addition of exogenous S1P attenuated the anti-tumor activity of carfilzomib at 5 and $10 \mathrm{nM}$. In particular, $10 \mathrm{nM}$ carfilzomib reduced cell growth by $72 \%$, while S1P addition resulted in only a $45 \%$ reduction. However, the anti-tumor effect of carfilzomib in the presence of S1P was restored when used in combination with fingolimod, SKI-I, or ABC294640 (Fig. 5B). In each bar plot of Fig. 5A-B, the mean and SD of three independent replicates are shown $(\mathrm{P}<0.05)$.

Anti-S1P agents inhibit S1P-promoted migration of HUVECs. We next evaluated the chemotactic response of HUVECs to S1P or its inhibitors. We observed that the addition of the supernatant of MM cells significantly induced HUVEC migration (Fig. 5C). This was probably S1P dependent, as incubation with S1P for $4 \mathrm{~h}$ significantly induced the migration of HUVECs compared with the control medium (with a peak effect detected at $100 \mathrm{nM} \mathrm{S1P)}$. However, simultaneous treatment with fingolimod, SKI-I, or ABC294640 inhibited S1P-induced cell migration (Fig. 5D; $\mathrm{P}<0.05$ ). Moreover, we observed increases in pMAPK and ERK1 within 5-15 min after adding S1P, followed by decreased pMAPK thereafter (until $60 \mathrm{~min}$ ) (Fig. 5E). The addition of the three anti-S1P agents repressed S1P-induced MAPK phosphorylation (Fig. 5F).

\section{Discussion}

In this study, we assessed the serum S1P levels among patients with MM or MGUS compared with those in healthy individuals. Notably, our results show that serum S1P levels were significantly higher among patients with MM than in healthy individuals.

S1P and SKs are involved in numerous cancer types, influencing cell growth, cell survival, mortality, transformation, and chemotherapy resistance $(7,10,16,17)$. However, although some recent studies suggested that S1P and SKs are involved in MM, their association with MM is unclear. For example, Yasui et al (21) reported that fingolimod has anti-cancer effects in MM cell lines. In addition, S1P may play important roles in the adhesion of MM cells, which is dependent on the $\alpha 4 \beta 1$ integrin (22). Venkata et al (23) reported that SK2 is overexpressed in both MM cell lines and primary cells and demonstrated the efficacy of SK2 inhibitors in inhibiting cell growth. Thus, further assessment of the roles of S1P and SKs in $\mathrm{MM}$ cell proliferation may facilitate the development of new treatment strategies for MM.

Xia et al (16) reported that SK1 activation, which depends on V12 RAS, promotes NIH/3T3 fibroblast transformation to fibrosarcoma, suggesting that SK1 has oncogenic activity. Thus, S1P activation via SKs is potentially associated with MM progression. The median serum S1P level among MM patients was higher than that of healthy volunteers. Our study is the first to report serum S1P elevation in MM patients. The proliferation of MM cells might be associated with S1P-related signaling. Anemia in MM is typically observed when the tumor is abundant, or the disease is advanced (24). The S1P level of MM patients with anemia were lower than that of those without anemia. The main supplier of serum S1P is red blood cells (RBC), and thus the low level of S1P in MM patients with anemia might be related to a reduction in $\mathrm{RBC}$. 
A

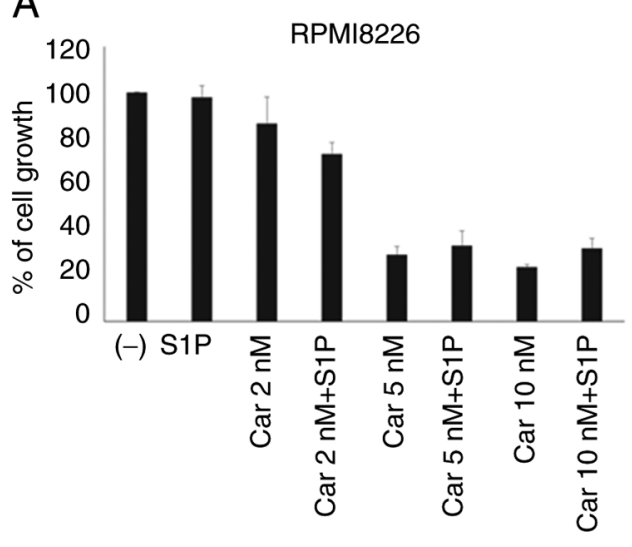

C

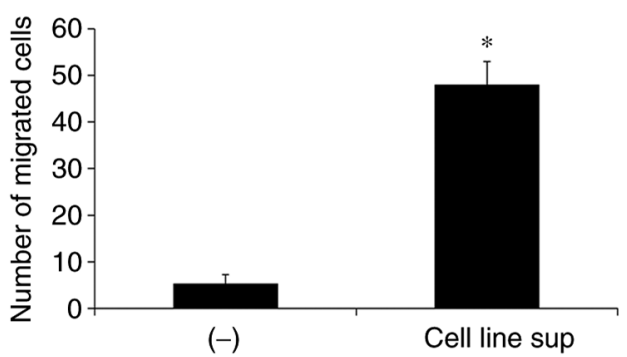

E

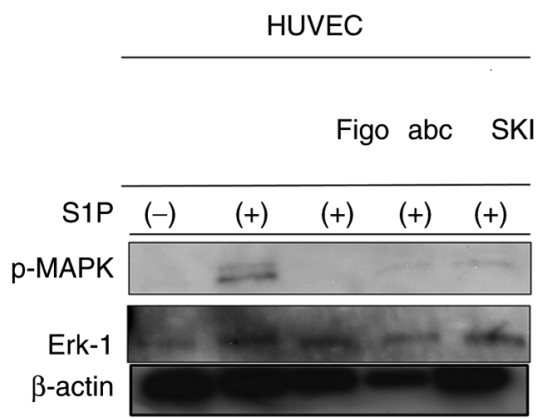

B

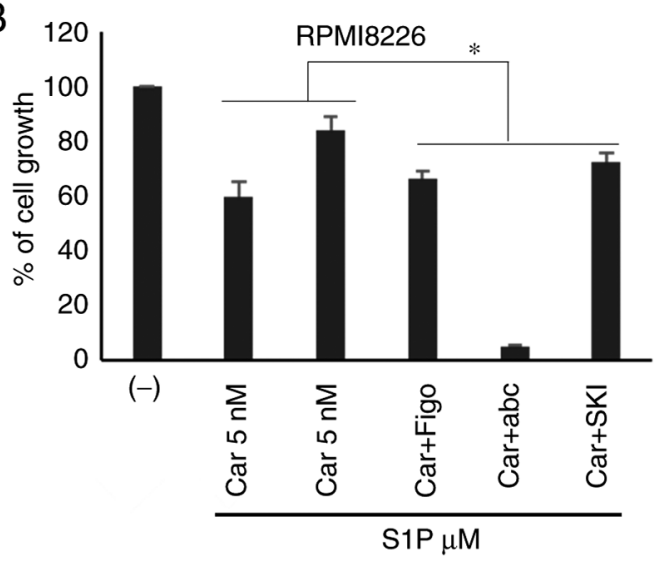

D

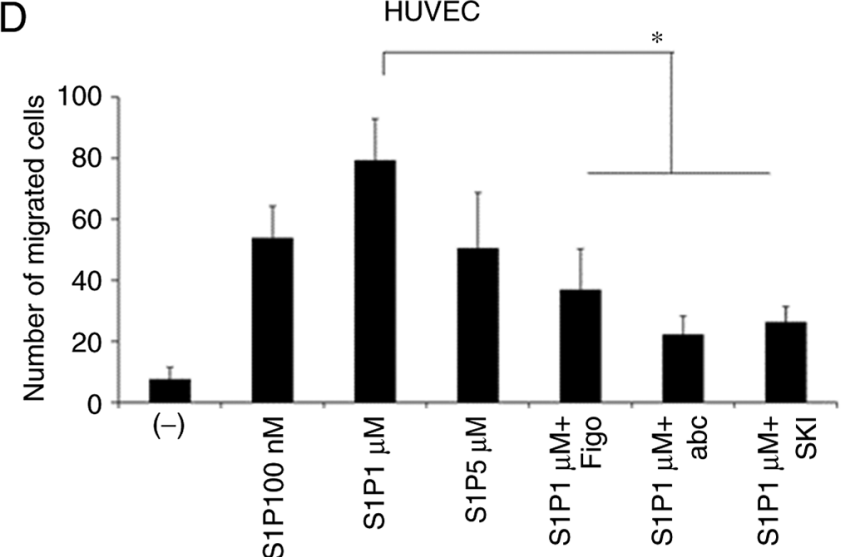

F

HUVEC

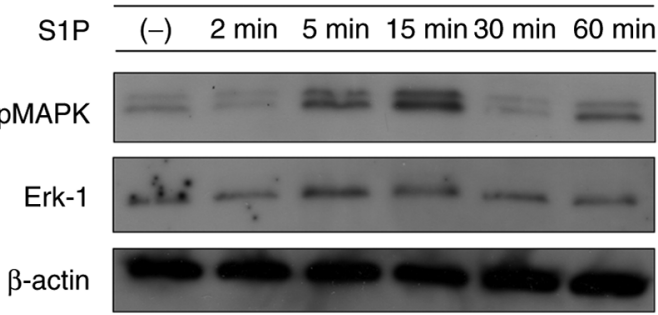

Figure 5. Cellular response of MM cell lines and HUVECs treated with carfilzomib, S1P and anti-S1P agents. (A) Effect of exogenous S1P on RPMI8226 cell growth inhibition by carfilzomib. (B) Effect of S1P addition during treatment with carfilzomib and the indicated inhibitor in different MM cell lines. ${ }^{*} \mathrm{P}<0.05$. (C) Migration assay of HUVECs in the presence of a supernatant derived from a MM cell line. ${ }^{*} \mathrm{P}<0.05$ cell line supernant vs. control. (D) Migration assay assessing the effect of S1P inhibitors on the S1P-mediated migration of HUVECs. " $\mathrm{P}<0.05$ vs. S1P1 $1 \mu \mathrm{M}$. (E) Immunoblot assay showing the expression kinetic of pMAPK and ERK1 in HUVECs upon S1P addition. (F) Immunoblot assay showing the effect of S1P inhibitors on the expression of pMAPK. ABC, ABC294640; car, carfilzomib; Figo, fingolimod; HUVECs, human umbilical vessel cells; MM, multiple myeloma; p-, phosphorylated; S1P, sphingosine 1-phosphate; SK, sphingosine kinase.

However, the median S1P level of MM patients with anemia were higher than that of healthy volunteers, indicating that S1P level might be constantly high among MM patients.

This study showed that the addition of exogenous S1P reduced the efficacy of PI in the RPMI8226 myeloma cell line, suggesting that S1P is involved in increased PI resistance in MM cells. These results show that the tested anti-S1P agents enhanced the PI-dependent cytotoxic effect in MM cell lines and primary myeloma cells, even in the presence of S1P, which can reduce PI-dependent antimyeloma effects. Taken together, these findings suggest the involvement of S1P in regulating proteasome activity. Moreover, some studies have reported associations among SK1, S1P, and the ubiquitinproteasome system (UPS). For instance, S1P promotes
NF- $\kappa \mathrm{B}$ activation by interacting with the $\mathrm{E} 3$ ubiquitin ligase TNF receptor-associated factor 2 as a cofactor (25). This interaction activates proteasome and induces inflammation. Other studies have reported that S1P accumulation leads to UPS activation owing to the concomitant downregulation of the deubiquitinating enzyme $(26,27)$. Accordingly, myeloma cell proliferation may be promoted by the upregulation of S1P and SKs through increased UPS activation. Therefore, inhibition of S1P signaling may suppress UPS and increase the efficacy of PI. S1P signaling is also involved in other signaling pathways. S1P potentially activates the phosphatidylinositol 3-kinase (PI3K)/protein kinase B (AKT)/mammalian target of rapamycin (mTOR) pathway through S1PR1 (28). When MM cells were cultured with an 
anti-S1P agent, alone or in combination with carfilzomib, we observed a stronger reduction in pS6 than with carfilzomib monotherapy. Furthermore, PI3K signaling is very active in myeloma cells (29). Therefore, inhibition of S1P-mediated MM cell proliferation may be PI3K signaling-dependent. Moreover, all the tested anti-S1P agents promoted apoptosis in MM cells by increasing the levels of cleaved caspase-3 and PARP. Thus, our results suggest that the tested inhibitors promote caspase-induced apoptosis and suppress UPS and PI3K signaling by inactivating the S6 ribosomal protein, thereby repressing translation.

The bone marrow microenvironment plays an important role in the pathophysiology and progression of MM. In particular, angiogenesis is strongly associated with myeloma cell proliferation (30). In vascular endothelial cells, S1P regulates proliferation, migration, and angiogenesis. This study shows that the inhibition of S1P activity by fingolimod or SK inhibitors suppressed the migration of HUVECs. Therefore, the use of these inhibitors potentially reduces S1P-mediated angiogenesis in the bone marrow microenvironment. Similarly, LaMontagne et al (11) reported that fingolimod suppressed tumor angiogenesis and proliferation in a mouse model. We observed that upon culturing HUVECs with S1P, the levels of pMAPK and ERK-1 increased. However, the expression levels reverted to baseline upon treatment with S1P antagonists. These results suggest that S1P signaling affects angiogenesis by modulating endothelial cell migration and proliferation through the MAPK signaling pathway.

Overall, our results indicate that inactivation of S1P by an S1PR1 antagonist and two SK inhibitors affected MM cell growth and apoptosis. In addition, these inhibitors displayed synergistic effects with PI carfilzomib treatment, even in the presence of S1P-mediated resistance. Moreover, inhibition of S1PR1 and SKs impaired the migration of endothelial cells, which is a critical mechanism involved in angiogenesis in the bone marrow microenvironment.

In conclusion, the results of the present study suggest that combinatorial treatment of PI with fingolimod or SK inhibitors constitutes a novel approach to treat MM and to overcome chemotherapeutic resistance. However, it is uncertain whether these agents can be developed as new therapeutic drugs for MM. Even though this study is limited by its small sample size and use of only in vitro data, the identified association between myeloma cell growth and S1P signaling is a new finding and might be helpful in decision making when choosing existing myeloma drugs and biomarkers for disease prognosis.

\section{Acknowledgements}

The authors would like to thank Miss Sayaka Ohmura and Dr Tomohiro Umezu (Tokyo Medical University, Division of Hematology) for providing technical support.

\section{Funding}

This work was supported by grants-in-aid for Scientific Research from the Ministry of Education, Culture, Sports Science and Technology (MEXT; grant no. 24701036) and the Support Centre of Doctor, Student and Researcher, Tokyo Medical University.

\section{Availability of data and materials}

The datasets used and/or analyzed are available from the corresponding author on reasonable request.

\section{Authors' contributions}

YT and SO designed the research, performed the experiments, confirmed the authenticity of all the raw data and wrote the manuscript. SO, YT, KO and AG developed the methodology. All authors read and approved the final manuscript.

\section{Ethics approval and consent to participate}

This research was approved by the Ethics Committee of Tokyo Medical University (approval ID, SH24081; March 3, 2012).

\section{Patient consent for publication}

Not applicable.

\section{Competing interest}

The authors declare that they have no competing interests.

\section{References}

1. Kuehl WM and Bergsagel PL: Molecular pathogenesis of multiple myeloma and its premalignant precursor. J Clin Invest 122: 3456-3463, 2012

2. Kyle RA and Rajkumar SV: An overview of the progress in the treatment of multiple myeloma. Expert Rev Hematol 7: 5-7, 2014.

3. Nooka AK, Kastritis E, Dimopoulos MA and Lonial S: Treatment options for relapsed and refractory multiple myeloma. Blood 125: 3085-3099, 2015.

4. Pyne S and Pyne NJ: Sphingosine 1-phosphate signaling in mammalian cells. Biochem J 349: 385-402, 2000.

5. Spiegel S and Milstein S: Sphingosine 1-phosphate: An enigmatic signaling lipid. Nat Rev Mol Cell Biol 4: 397-407, 2003.

6. Hannun YA and Obeid LM: Principles of bioactive lipids signaling: Lessons from sphingolipids. Nat Rev Mol Biol 9: 139-150, 2008.

7. Pyne NJ, El Buri A, Adams DR and Pyne S: Sphingosine 1-phosphate and cancer. Adv Biol Regul 68: 97-106, 2008.

8. Ogretmen B and Hannun YA: Biologically active sphingolipids in cancer pathogenesis and treatment. Nat Rev Cancer 4: 604-616, 2004.

9. Pyne S, Lee SC, Long J and Pyne NJ: Role of sphingosine kinases and lipid phosphate phosphatases in regulating spatial sphingosine 1-phosphate signalling in health and disease. Cell Signal 21: 14-21, 2009

10. Pyne NJ and Pyne S: Sphingosine 1-phosphate and cancer. Nat Rev Cancer 10: 489-503, 2010.

11. LaMontagne K, Littlewood-Evans A, Schnell C, O'Reilly T, Wyder L, Sanchez T, Probst B, Butler J, Wood A, Liau G, et al: Antagonist of sphingosine 1-phosphate receptors by FTY720 inhibits anginogenesis and tumor vascularization. Cancer Res 66: 221-231, 2006.

12. Paugh SW, Paugh BS, Rahmani M, Kapitonov D, Almenara JA, Kordula T, Milstien S, Adams JK, Zipkin RE, Grant S and Spiegel S: A selective sphingosine kinasel inhibitor integrates multiple molecular therapeutic targets in human leukemia. Blood 112: 1382-1391, 2008.

13. French KJ, Zhuang Y, Maines LW, Gao P, Wang W, Beljanski V, Upson JJ, Green CL, Keller SN and Smith CD: Pharmacology and antitumor activity of ABC294640, a selective inhibitor of sphingosine kinase-2. J Pharm Exp Ther 333: 129-139, 2010.

14. Beljanski V, Knaak C and Smith CD: A novel sphingosine kinase inhibitor induced autophagy in tumor cells. J Pharm Exp Ther 333: 454-464, 2010.

15. Neubauer HA and Pitson SM: Roles, regulation and inhibitors of sphingosine kinase 2. FEBS J 280: 5317-5336, 2013. 
16. Xia P, Gamble JR, Wang L, Pitson SM, Moretti PA, Wattenberg BW, D'Andrea RJ and Vadas MA: An oncogenic role of sphingosine kinase. Curr Biol 10: 1527-1530, 2000.

17. Akao Y, Banno Y, Nakagawa Y, Hasegawa N, Kim TJ, Murate T, Igarashi $\mathrm{Y}$ and Nozawa $\mathrm{Y}$ : High expression of sphingosine kinase 1 and S1P receptors in chemotherapy-resistant prostate cancer PC-3 cells and their camptothecin-induced up-regulation. Biochem Biophys Res Commun 342: 1284-1290, 2006.

18. Okabe S, Tanaka Y, Tauchi T and Ohyashiki K: Copanlisib, a novel phosphoinositide 3-kinase inhibitor, combined with carfilzomib inhibits multiple myeloma cell proliferation. Ann Hematol 98: 723-733, 2019.

19. Chen HC: Boyden chamber assay. Methods Mol Biol 294: 15-22, 2005.

20. Ishii I, Fukushima N, Ye X and Chun J: Lysophospholipid receptors: Signaling and biology. Annu Rev Biochem 73: 321-354, 2004.

21. Yasui H, Hideshima T, Raje N, Roccaro AM, Shiraishi N, Kumar S, Hamasaki M, Ishitsuka K, Tai YT, Podar K, et al: FTY720 induces apoptosis in multiple myeloma cells and overcomes drug resistance. Cancer Res 65: 7478-7484, 2005.

22. Sanz-Rodríguez F, Hidalgo A and Teixidó J: Chemokine stromal cell-derived factor- $1 \alpha$ modulates VLA-4 integrin-mediated multiple myeloma cell adhesion to CS-1/fibronectin and VCAM-1. Blood 97: 346-351, 2001.

23. Venkata JK, An N, Stuart R, Costa LJ, Cai H, Coker W, Song JH, Gibbs K, Matson T, Garrett-Mayer E, et al: Inhibition of sphingosine kinase 2 downregulates the expression of c-Myc and Mcl-1 and induces apoptosis in multiple myeloma. Blood 124: 1915-1925, 2014.

24. Durie BG and Salmon SE: A clinical staging system for multiple myeloma. Correlation of measured myeloma cell mass with presenting clinical features, response to treatment, and survival. Cancer 36: 842-854, 1975.
25. Alvarez SE, Harikumar KB, Hait NC, Allegood J, Strub GM, Kim EY, Maceyka M, Jiang H, Luo C, Kordula T, et al: Sphingosine-1-phosphate is a missing cofactor for the E3 ubiquitin ligase TRAF2. Nature 465: 1084-1088, 2010.

26. Mitroi DN, Deutschmann AU, Raucamp M, Karunakaran I, Glebov K, Hans M, Walter J, Saba J, Gräler M, Ehninger D, et al: Sphingosine 1-phosphate lyase ablation disrupts presynaptic architecture and function via ubiquitin-proteasome mediated mechanism. Sci Rep 6: 1-15, 2016.

27. Wallington-Beddoe CT, Bennett MK, Vandyke K, Davies L, Zebol JR, Moretti PA, Pitman MR, Hewett DR, Zannettino AC and Pitson SM: Sphingosine kinase 2 inhibition synergises with bortezomib to target myeloma by enhancing endoplasmic reticulum stress. Oncotarget 8: 43602-42616, 2017.

28. Banno Y, Takuwa Y, Akao Y, Okamoto H, Osawa Y, Naganawa T, Nakashima S, Suh PG and Nozawa Y: Involvement of phospholipase D in sphingosine 1-phosphate-induced activation of phosphatidylinositol 3-kinase and Akt in Chinese hamster ovary cells overexpressing EDG3. J Biol Chem 276: 35622-35628, 2001.

29. Ikeda H, Hideshima T, Fulciniti M, Perrone G, Miura N, Yasui H, Okawa Y, Kiziltepe T, Santo L, Vallet S, et al: PI3K/p110 is a novel therapeutic target in multiple myeloma. Blood 116: 1460-1468, 2010.

30. Kumar S, Fonseca R, Dispenzieri A, Lacy MQ, Lust JA, Wellik L, Witzig TE, Gertz MA, Kyle RA, Greipp PR and Rajkumar SV: Prognostic value of angiogenesis in solitary bone plasmacytoma. Blood 101: 1715-1717, 2003.

This work is licensed under a Creative Commons Attribution-NonCommercial-NoDerivatives 4.0 International (CC BY-NC-ND 4.0) License. 